\title{
Miniature camera lens design with a freeform surface
}

\section{Yufeng Yan, Jose Sasian}

Yufeng Yan, Jose Sasian, "Miniature camera lens design with a freeform surface," Proc. SPIE 10590, International Optical Design Conference 2017, 1059012 (27 November 2017); doi: 10.1117/12.2292653

SPIE Event: International Optical Design Conference - IODC 2017, 2017, Denver, United States 


\title{
Miniature Camera Lens Design with a Freeform Surface
}

\author{
Yufeng Yan*, Jose Sasian \\ University of Arizona, College of Optical Sciences, 1630 East University Boulevard, Tucson, \\ Arizona, 85721
}

\begin{abstract}
We present a miniature camera lens design method that uses a freeform surface based on the pedal curve to the ellipse in polynomial form. Two designs are presented and their benefits of optical performance and tolerance sensitivity are compared to designs with conventional aspheric surfaces. We also reverse a freeform design using even aspherical surfaces to show that the optimization solution of a freeform design cannot be reproduced by even aspherical surfaces.
\end{abstract}

Keywords: Lens design, freeform surface, lens tolerance, miniature camera

\section{INTRODUCTION}

Camera lens designs for mobile platform electronics applications, such as cell phones and tablets, have been rapidly developed in the past decade. Although these miniature cameras in mobile devices are becoming ubiquitous in our daily lives, better optical performance is always demanded. To achieve good optical performance in these miniature cameras, aspherical surfaces are extensively used during the lens design, usually employing up to 16th order terms. However, the performance of the miniature cameras designed with conventional aspherical surfaces is approaching a limit. While lens designers are still pushing the limits of their designs with conventional even/odd aspherical surfaces, a more efficient surface description is desirable for improvement. A recently published paper ${ }^{1}$ introduced a freeform surface that combines base surfaces of the pedal curve to the ellipse for light illumination control. In this paper, we discuss the benefits of using such pedal curve and its freeform combination for miniature camera lens optimization. Section 2 briefly explains some design challenges of miniature camera lenses. Section 3 discusses the form of pedal curve to the ellipse and its freeform combination. In section 4, we optimize a patented lens with both even aspheric surfaces and the pedal curve polynomial, and the performance comparison and tolerance analysis are provided. In section 5, we optimized another patented lens using Q-type polynomial surfaces along with the conventional even asphere, and the pedal polynomial surface. Then tolerance sensitivity and polynomial coefficient number are compared. In section 6, we replaced the pedal surfaces from the lens in section 5 back to even aspherical surfaces to make a 'reversed even asphere lens' and then evaluate its performance.

\section{CHALLENGES OF MINIATURE CAMERA LENSES}

When designing miniature camera lenses, lens designers are facing great challenges compared to designing conventional large-scale camera lenses. The most limiting specification is the package size. In order to avoid color crosstalk on the digital sensor, the image space chief ray angle (CRA) is limited, usually to no more than 30 degrees $^{2}$. Thus the stop aperture must be located close to the first surface to fulfil the CRA requirement, which causes the lens to be non-symmetric about the stop. The lack of symmetry about the stop makes correcting distortion and lateral color difficult. In addition, miniature camera lenses have been using injection molding of plastic instead of conventional glasses. The reduced choice of materials and the fact that plastic doublets are difficult to manufacture also challenge lens designers on controlling chromatic aberration and field curvature. Due to these challenges in miniature lens design, many design techniques for designing conventional camera lenses cannot be applied, and the structure of a miniature camera lens becomes different compared to a conventional camera lens. To efficiently correct aberrations in a miniature camera, lens designers depend on the extensive use of aspherical surfaces. Currently, five lens element designs are widely used by mobile device companies. However, due to the demand of low-light performance, large aperture lenses with lower F/\# are desired. More lens elements may be needed and this makes it difficult to maintain the total track length (TTL). At the same time, mobile devices are becoming thinner, which causes the lens to protrude over the surface of some mobile devices. Furthermore, to ensure

International Optical Design Conference 2017, edited by Peter P. Clark, Julius A. Muschaweck, Richard N. Pfisterer, John R. Rogers, Proc. of SPIE-OSA Vol. 10590, 1059012 · @ 2017 SPIE CCC code: $0277-786 \mathrm{X} / 17 / \$ 18 \cdot$ doi: $10.1117 / 12.2292653$ 
uniformity of the image, the relative illumination (RI) is often required to be maintained at least to $50 \%$ at the sensor corners $^{3}$. Nevertheless, there is a tradeoff between relative illumination and aberration control during the lens optimization.

\section{PEDAL SURFACE POLYNOMIAL}

In miniature camera lens design, the rear group usually contains one or two elements that are strongly aspheric to efficiently correct field curvature, astigmatism and distortion ${ }^{4}$. The shape of these elements cannot be easily explained by conventional aberration theory, as the aspheres become dominant at large field angles ${ }^{2}$. However, by reviewing current lens patents with 5 elements and 6 elements, one can realize that these strongly aspherical elements often contain surfaces with different curvature direction from the center of the surface to the edge (e.g., concave in the center and turning back to convex before the edge). Table 1 shows some examples of such surfaces from various mobile camera lens patents. It is noted that this kind of surface profile can be described by the pedal curve to the ellipse (Fig. 1). The equation for the pedal curve to the ellipse on the $\mathrm{x}-\mathrm{y}$ plane in a Cartesian coordinate system is

$$
a^{2} x^{2}+b^{2} y^{2}=\left(x^{2}+y^{2}\right)^{2}
$$

where $a$ is the major axis of the ellipse, $b$ is the minor axis, and $r$ is the radial distance from the optical axis. The sag $\mathrm{S}(\mathrm{r})$ of this pedal surface is obtained by rotation about the $\mathrm{z}$-axis

$$
S(r)=b-\sqrt{\frac{b^{2}-2 r^{2}+\sqrt{b^{4}+4\left(a^{2}-b^{2}\right) r^{2}}}{2}}
$$

A freeform polynomial surface ${ }^{1}$ can be written as a superposition of several pedal surfaces

$$
z_{p}(r)=A_{1} S_{1}(r)+A_{2} S_{1}^{2}(r)+A_{3} S_{1}^{3}(r)+B_{1} S_{2}(r)+B_{2} S_{2}^{2}(r)+B_{3} S_{2}^{3}(r)
$$

where $S_{1}(r)$ and $S_{2}(r)$ are two sets of pedal surfaces, $A_{1}-A_{3}$ and $B_{1}-B_{3}$ are coefficients.

Table 1. Some patent examples to show the strongly aspherical surfaces in the rear group of a miniature camera lens

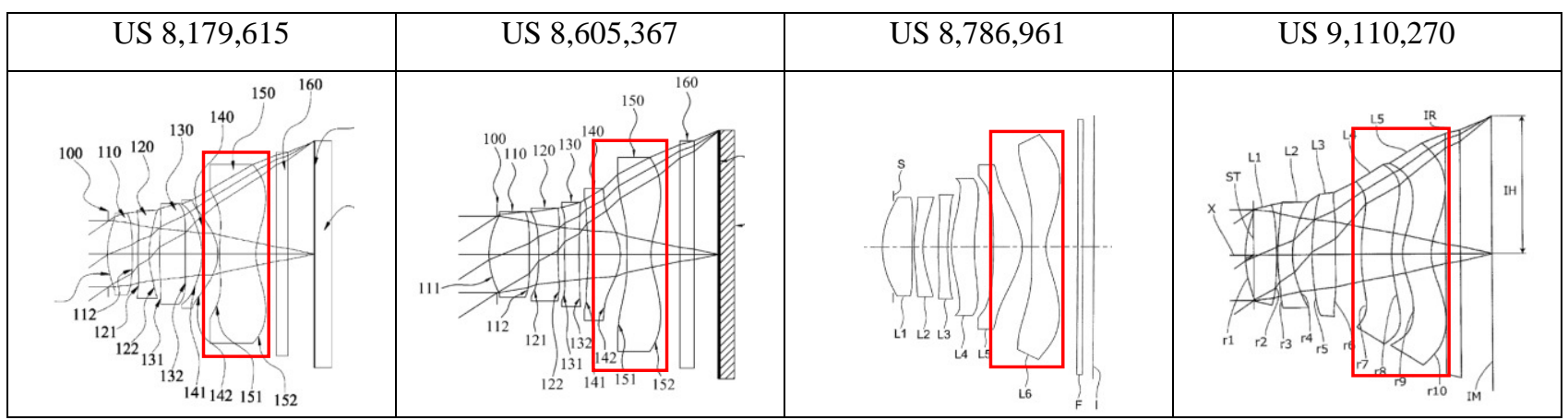

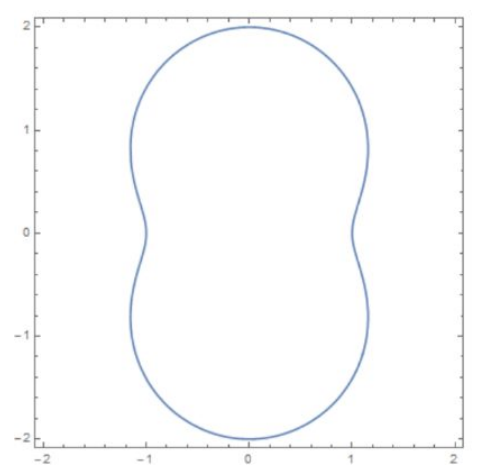

Fig. 1. An example pedal curve to the ellipse, $a=2, b=1$. 


\section{FIRST LENS COMPARISON}

\subsection{Benchmark lens and evaluation lens}

The starting point for a first benchmark lens design is from the first embodiment in U.S. Patent 9,110,270. The patent lens contains five lens elements with an IR filter in front of the sensor. The lens is re-optimized into our benchmark lens using only conventional even aspherical surfaces with the design specifications provided in Table 2. The sag $\mathrm{z}(\mathrm{r})$ of an even aspheric surface is

$$
z(r)=\frac{c r^{2}}{1+\sqrt{1-(1+k) c^{2} r^{2}}}+A_{2} r^{2}+A_{4} r^{4}+A_{6} r^{6}+A_{8} r^{8}
$$

where $\mathrm{c}$ is the vertex curvature of the surface, $\mathrm{k}$ is the conic constant, $\mathrm{r}$ is the radial distance from the optical axis, and aspheric coefficients are denoted as $\mathrm{A}_{2}, \mathrm{~A}_{4}, \mathrm{~A}_{6}$ and $\mathrm{A}_{8}$. The number of aspheric coefficients for each surface remains the same in the patent specification. This optimization is not intended to change the patent lens construction but rather to fix the errors in the lens patent data.

Table 2. Design specification for the first benchmark lens and evaluation lens

\begin{tabular}{|c|c|c|c|c|c|c|c|}
\hline Wavelength & $f[\mathrm{~mm}]$ & F/\# & FOV [deg] & TTL $[\mathrm{mm}]$ & Distortion & CRA [deg] & Edge RI \\
\hline g, F, d, C & 4.1 & 2.2 & 69.8 & $<5.2$ & $<0.5 \%$ & $<30$ & $>50 \%$ \\
\hline
\end{tabular}

Our evaluation lens uses the pedal polynomial freeform surface described in section 3 to replace surfaces $7,8,9$ and 10 in the rear lens group. The evaluation lens design uses the same specification as the benchmark lens. The same merit function and optimization procedures were used to ensure an un-biased lens comparison.

\subsection{Nominal performance comparison}

The layouts and the optical path difference (OPD) plots of both benchmark and evaluation lenses are shown in Fig. 2. The freeform surfaces are marked on the evaluation lens. The lens construction differences are minimal. On the performance side, the evaluation lens with the freeform surface provides a smoother OPD plot across the pupil and overall better aberration control. This better performance can also be observed in the MTF plot in Fig. 3. A more uniform contrast performance across the field of view is produced by the evaluation lens.
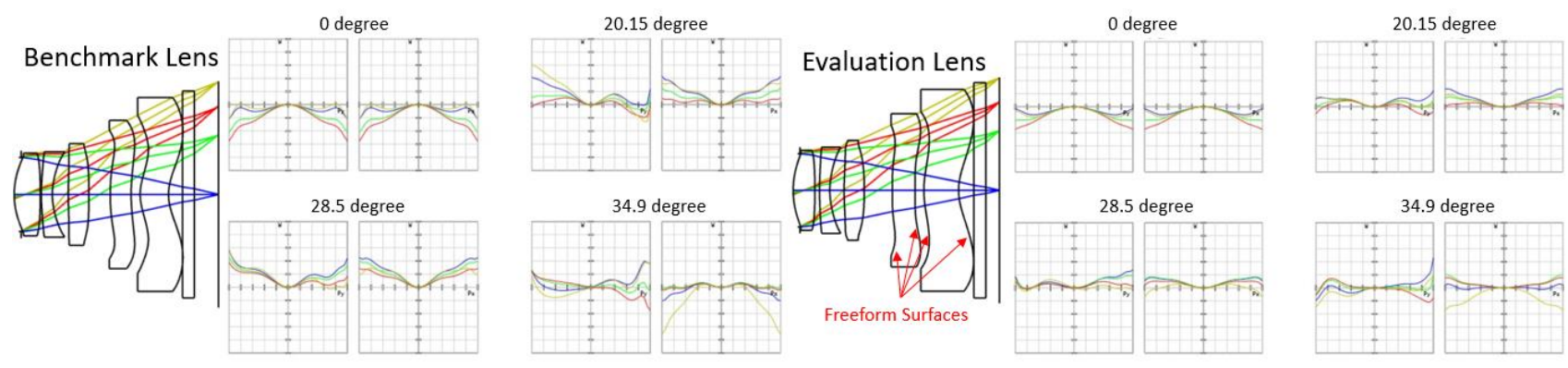

Fig. 2 Layouts and OPD plots for benchmark lens (left) and evaluation lens (right) 


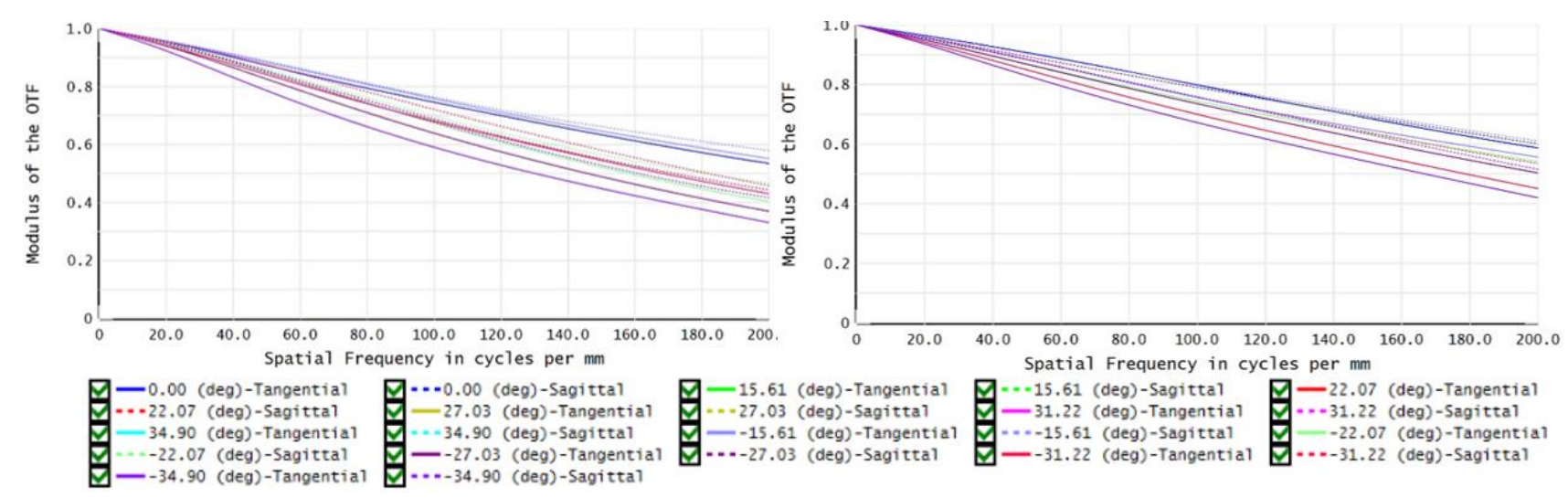

Fig. 3 Nominal MTF plots for benchmark lens (left) and evaluation lens (right)

\subsection{Tolerance sensitivity}

Other than the nominal optical performance, manufacturing and assembly errors are also a tolerancing challenge for lens designers. High order aspherical surfaces are usually sensitive to decenter and tilt as a single surface or as a part of a lens element. In this section, we test the surface and element decenter sensitivity on the rear lens elements for both designs. MTF plots are used for evaluation. Since the direction of decenter affects the result, we sample the field of view in both the positive $Y$ direction and the negative $Y$ direction in a single MTF plot to sufficiently analyze the decenter. Fig. 4 shows the MTF under a 5 micron surface decenter of the rear lens group for both lenses. The MTF plots under a 5 micron element decenter of the rear group are also presented in Fig. 5.

The MTF plots show that the benchmark lens suffers more from surface decenter than the evaluation lens. This difference in behavior comes from surfaces 7, 9 and 10. Surface 8 on both lenses is equally sensitive. Also, decenter of 4 th lens element has a significant impact on the benchmark lens, while the 5th lens element has a similar impact on both lenses. Overall, the evaluation lens with the freeform surfaces has less decenter tolerance sensitivity than the benchmark lens.

\section{SECOND COMPARISON}

\subsection{Comparison setup}

A second set of lenses are constructed to verify the result from the first lens comparison. The starting point for the second benchmark lens is from U.S. Patent 8,605,367. Using the same procedure as was used for the first benchmark lens, we reoptimized the patent lens with conventional even aspherical surfaces to fix minor errors in the patent data and keep our comparison un-biased. An evaluation lens is also re-optimized by replacing the rear group elements (surfaces 7, 8, 9 and 10) with pedal freeform surfaces. In addition, we set up another benchmark lens with Q-type polynomial surfaces. The Qtype polynomial surface is an alternate asphere type that was introduced in 2007 by G.W. Forbes ${ }^{6}$. An updated sag equation is given in a later paper in $2011^{7}$. According to Forbes, the Q-type polynomial surface provides faster manufacturability estimates and makes the design process more efficient. A previous paper also explains how a Q-type polynomial can potentially increase the yield of miniature camera lenses ${ }^{8}$. Thus, this benchmark lens with Q-type surfaces can serve as another reference to assess the performance of the evaluation lens. Up to 8 coefficients (including the conic constant) are used for the Q-type polynomial in the benchmark lens. Design specifications of these three lenses are provided in Table 3. 
0.00 (deg)-Tangential
-22.07 (deg) -Sagittal
-34.90 (deg)-Tangential
-22.07 (deg)-Sagittal $\square-34.90$ (deg)-Tangential $\square-22.07$ -
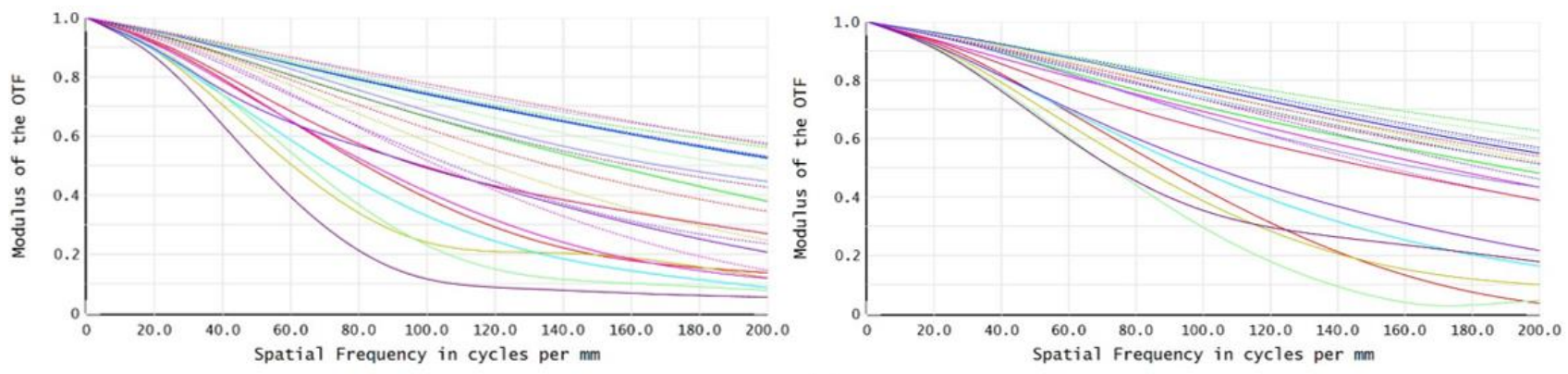

(a)
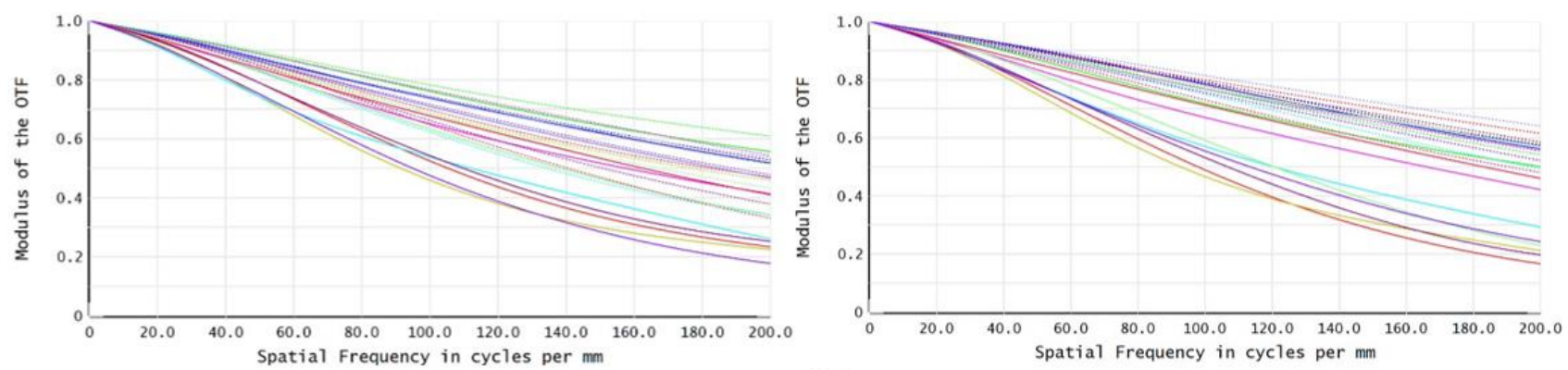

(b)
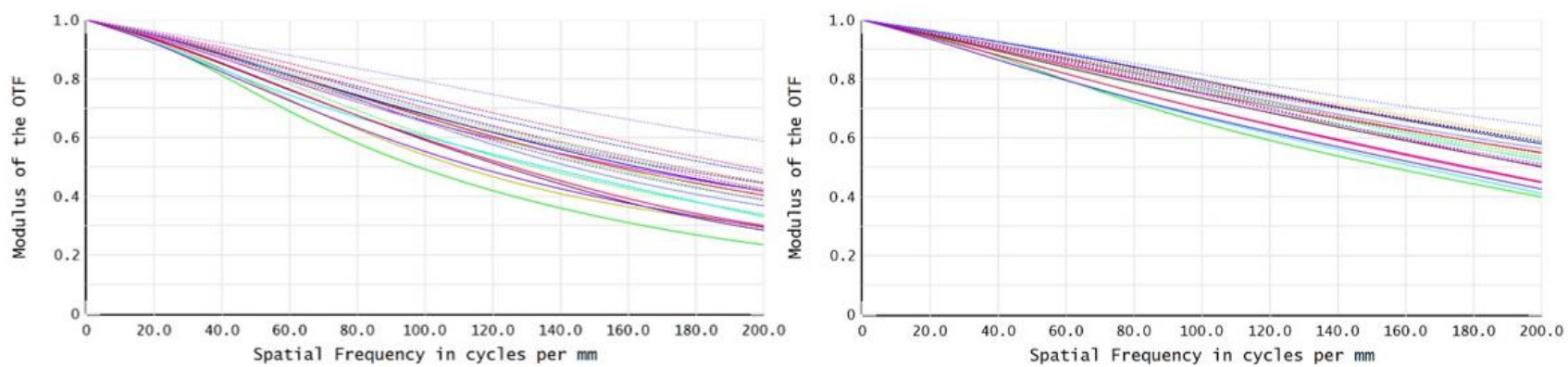

(c)
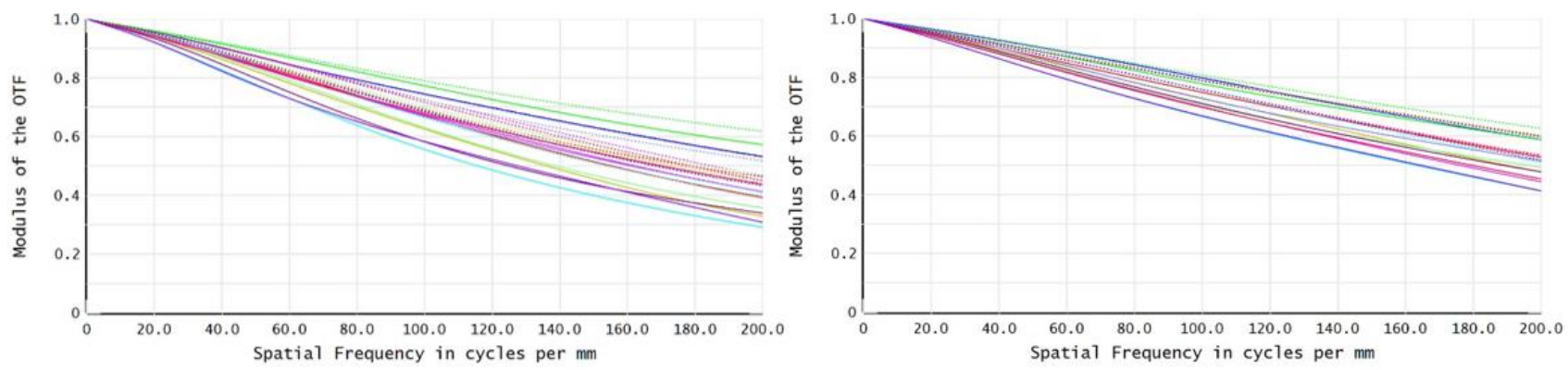

(d)

Fig. 4 MTF plots under 5 micron surface decenter of (a) surface 7, (b) surface 8, (c) surface 9 and (d) surface 10 for benchmark lens (left) and evaluation lens (right) 

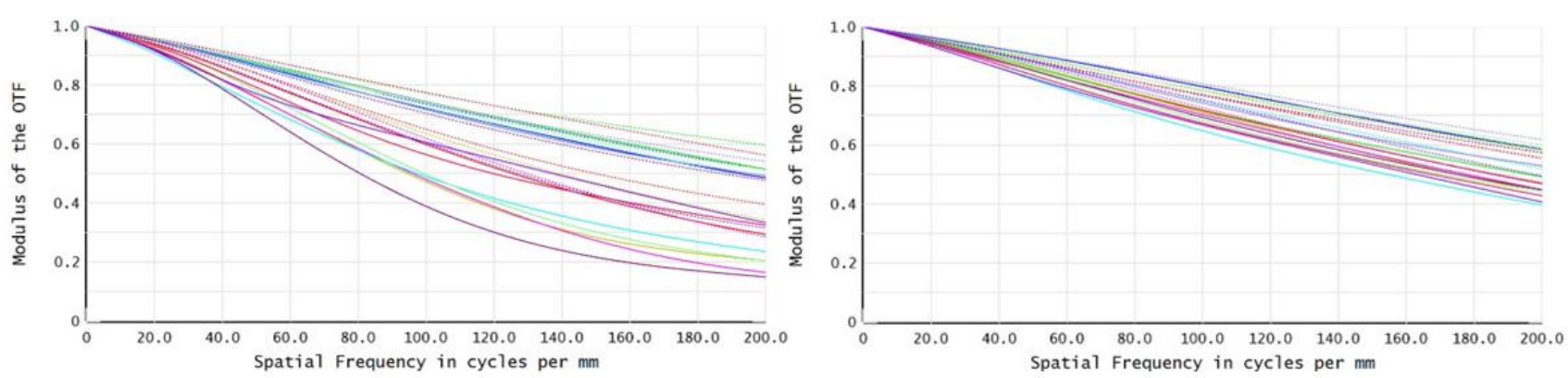

(a)
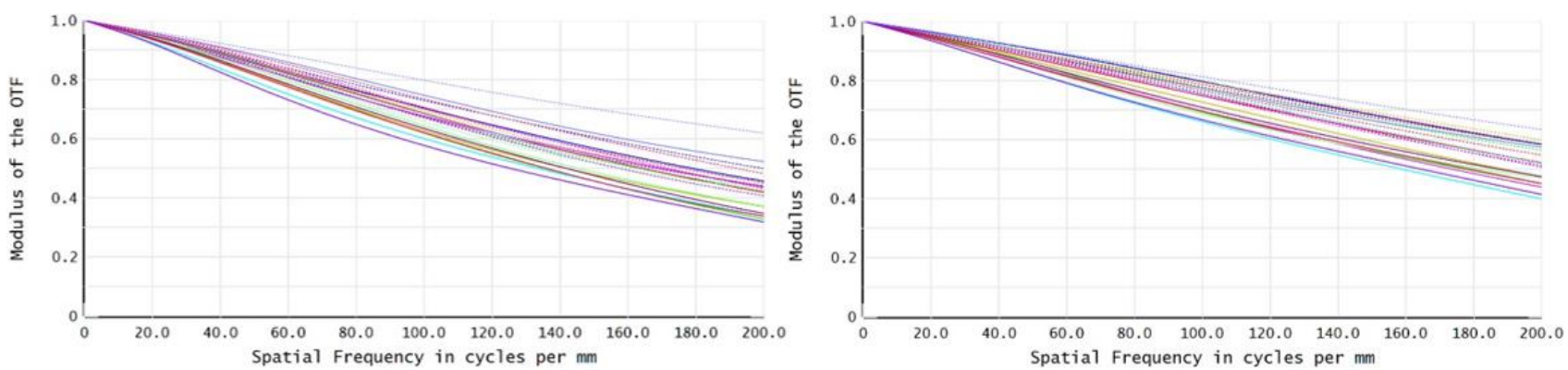

(b)

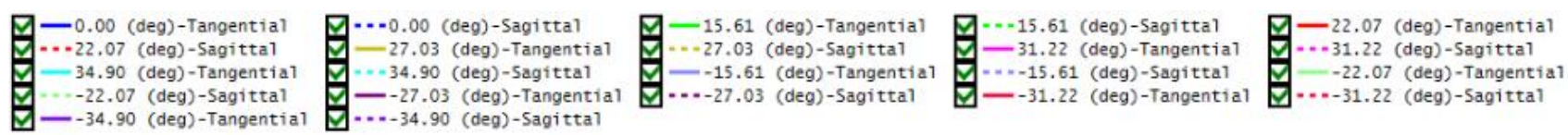

Fig. 5 MTF plots under 5 micron surface decenter of (a) element 4 and (b) element 5 for benchmark lens (left) and evaluation lens (right)

Table 3. Design specification for even asphere lens, Q-type polynomial lens, and pedal freeform lens

\begin{tabular}{|c|c|c|c|c|c|c|c|}
\hline Wavelength & $f[\mathrm{~mm}]$ & F/\# & FOV [deg] & TTL $[\mathrm{mm}]$ & Distortion & CRA [deg] & Edge RI \\
\hline $\mathrm{g}, \mathrm{F}, \mathrm{d}, \mathrm{C}$ & 1.9 & 2.4 & 67 & $<2.4$ & $<1 \%$ & $<30$ & $>50 \%$ \\
\hline
\end{tabular}

\subsection{Performance comparison}

Lens layouts for the even asphere lens, the Q-type polynomial lens, and the pedal freeform lens are shown in Fig. 6. The even asphere lens and the Q-type polynomial lens share similar construction, while the pedal freeform lens shows some surface shape change for the rear lens group. Fig. 7 shows the OPD plots for all three lenses. The pedal freeform lens continues to provide smoother and more uniform OPD across the pupil, but the overall aberration of theses lenses do not show significant differences. This can be confirmed by the MTF plots provided in Fig. 8. The contrast performance for the three lenses is almost identical.
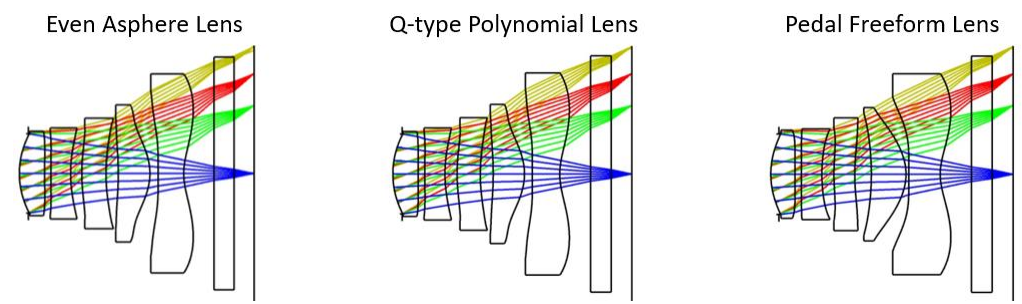

Fig. 6 Lens layout for even asphere lens (left), Q-type polynomial lens (middle) and pedal freeform lens (right). 


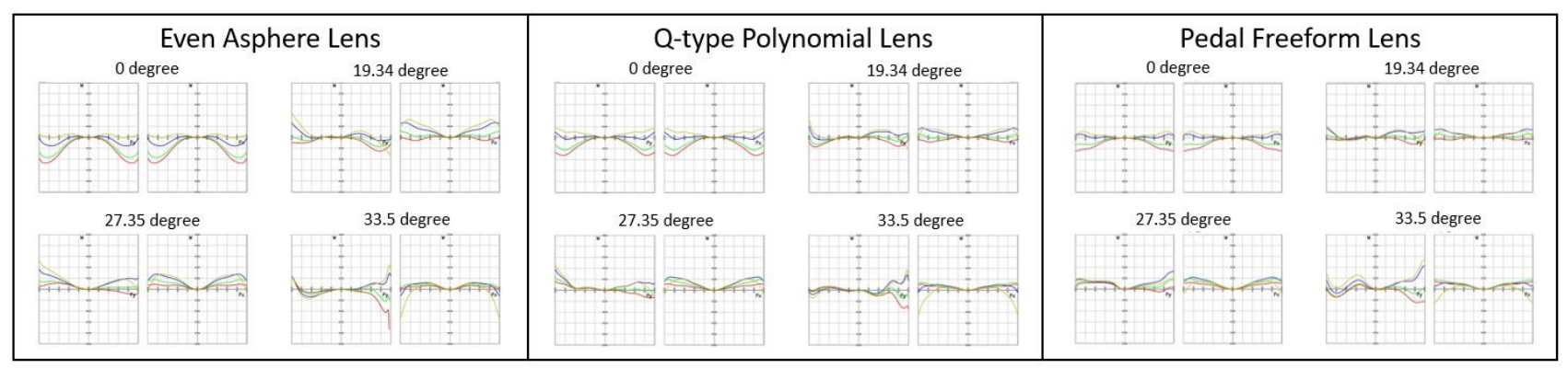

Fig. 7 Nominal OPD plots for even asphere lens (left), Q-type polynomial lens (middle) and pedal freeform lens (right).
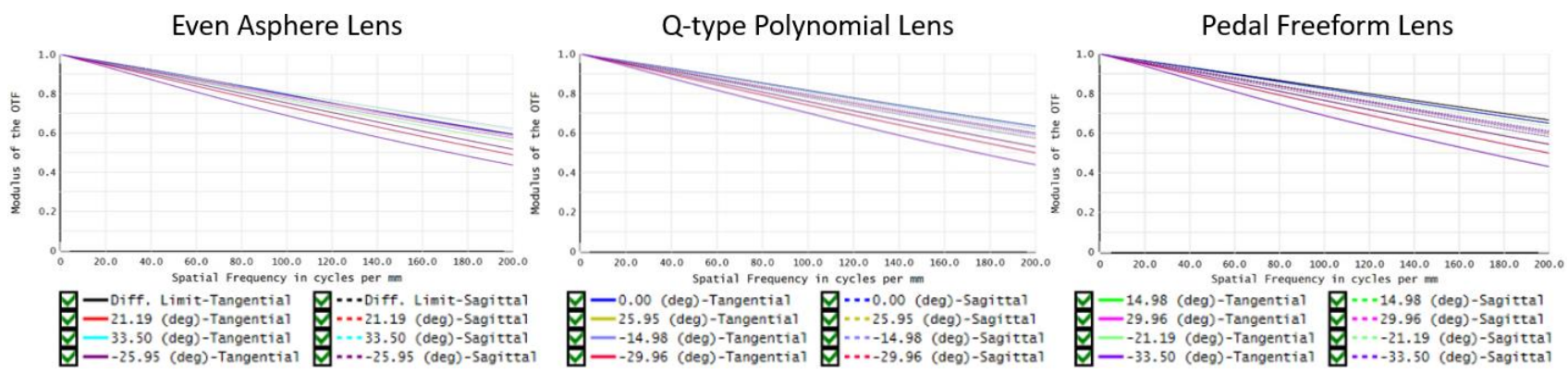

Fig. 8 Nominal MTF plots for even asphere lens (left), Q-type polynomial lens (middle) and pedal freeform lens (right).

While all the three lenses have similar nominal optical performance, the performance under the influence of surface and element decenter shows significant differences between the lenses. Fig. 9 and Fig. 10 show the MTF plots with a 5 micron element and surface decenter. The pedal freeform lens shows much lower sensitivity to decenter compared to the other two lenses. There is not much sensitivity difference between the even asphere lens and the Q-type polynomial lens.
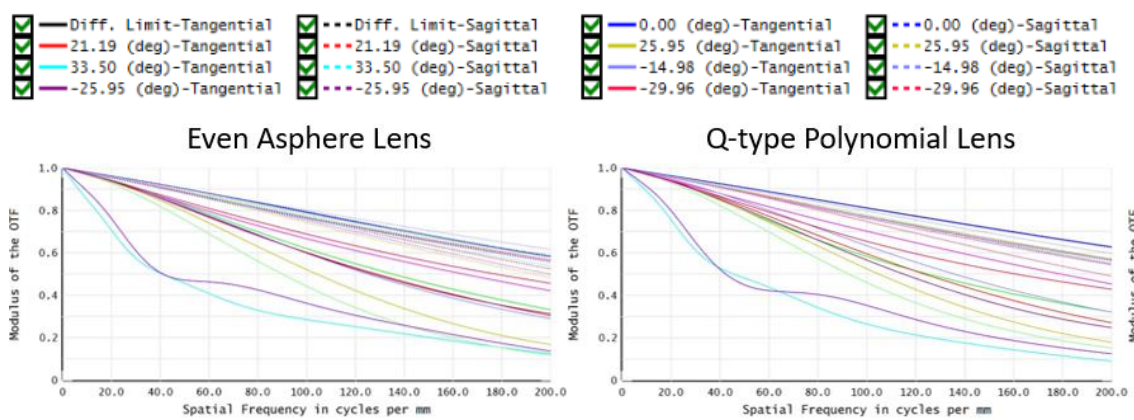

(a)
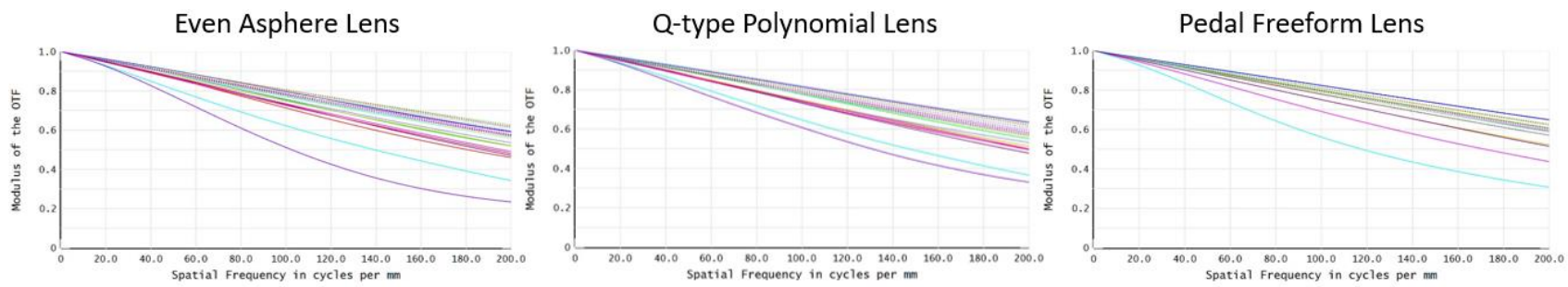

(b)

Fig. 9 MTF plots under 5 micron surface decenter of (a) element 4 and (b) element 5 for even asphere lens (left), Q-type polynomial lens (middle) and pedal freeform lens (right). 
D-Diff. Limit-Tangential - 33.50 (deg)-Tangential $y=-33.50$ (deg)-Sagittal - -25.95 (deg)-Tangential

Even Asphere Lens

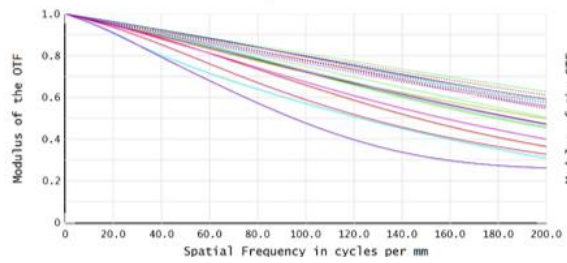

Even Asphere Lens

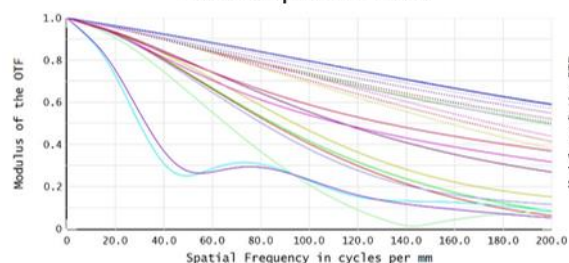

Even Asphere Lens
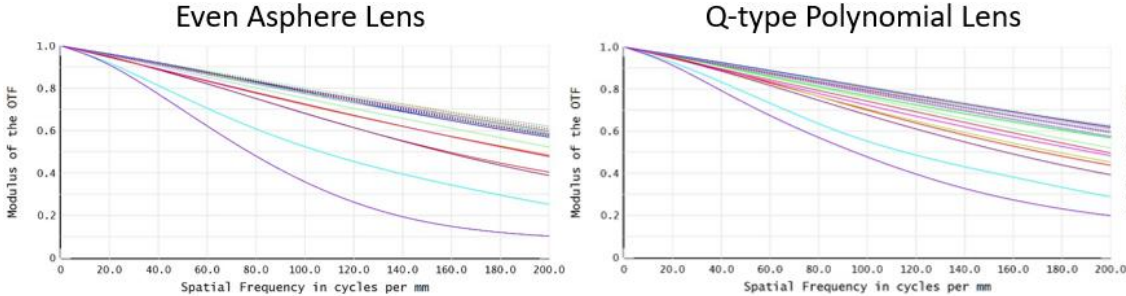

(c)
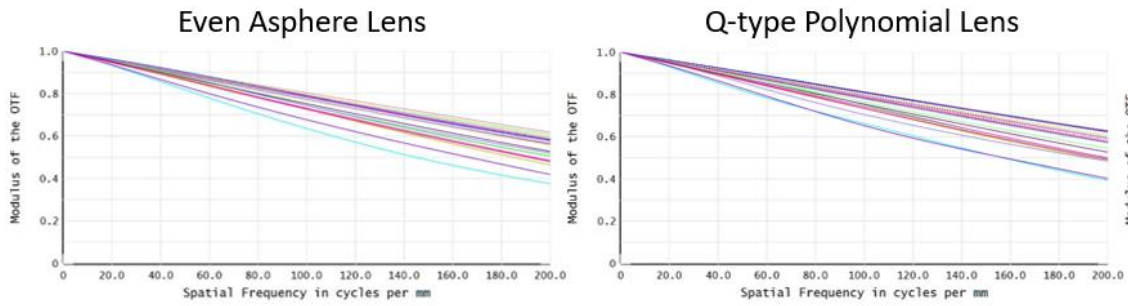

(d)

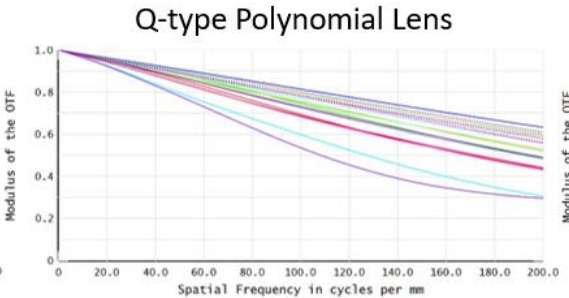

(a)

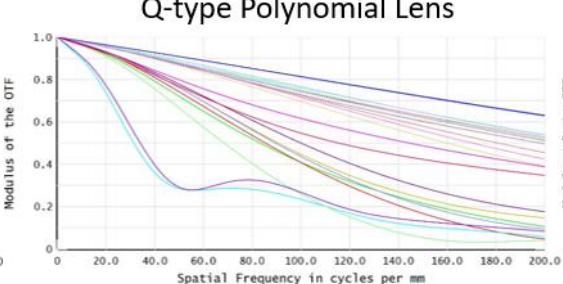

(b)

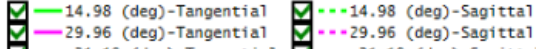
- -33.50 (deg)-Tangential
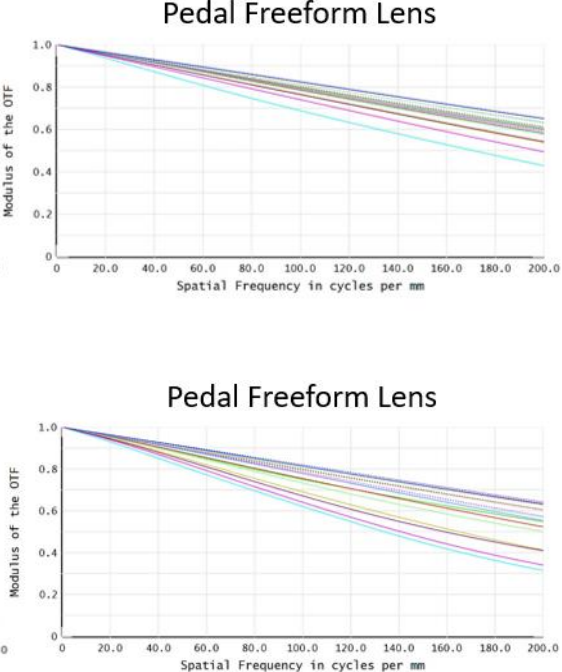

Fig. 10 MTF plots under 5 micron surface decenter of (a) surface 7, (b) surface 8, (c) surface 9 and (d) surface 10 for even asphere lens (left), Q-type polynomial lens (middle) and pedal freeform lens (right).

Another way to assess an aspherical/freeform design is by its total number of polynomial coefficients for each surface. While more coefficients provide more degrees of freedom, and usually benefit the optical performance, too many coefficients often appear to be redundant. Also, the extensive use of aspheric terms often leads to wiggles and unnecessary variation on a surface; the variations can add error rather than cancel error. Keeping the same optical performance while reducing the number of polynomial coefficients is usually a goal for lens designers. Less coefficients make the design cleaner and result in a more efficient design. Table 4 shows the number of polynomial coefficients, including the conic constant, of the surfaces in the rear lens group of each lens. The freeform design can achieve the same nominal performance and better sensitivity performance with less coefficients. 
Table 4. Number of polynomial coefficients, including the conic number, of all 3 lenses under investigation

\begin{tabular}{|c|c|c|c|}
\hline Surface & Even Asphere Lens & Q-type Polynomial Lens & Pedal Freeform Lens \\
\hline 7 & Up to $16^{\text {th }}$ order, 8 Coefficients & 8 Coefficients & 1 set of pedal polynomial, 3 coefficients \\
\hline 8 & Up to $16^{\text {th }}$ order, 8 Coefficients & 8 Coefficients & 1 set of pedal polynomial, 3 coefficients \\
\hline 9 & Up to $16^{\text {th }}$ order, 8 Coefficients & 8 Coefficients & 1 set of pedal polynomial, 3 coefficients \\
\hline 10 & Up to $16^{\text {th }}$ order, 8 Coefficients & 8 Coefficients & 2 sets of pedal polynomial, 6 coefficients \\
\hline
\end{tabular}

\section{REVERSED ASPHERE DESIGN}

In this section, we verify the less sensitive lens with pedal surfaces. Since this freeform design has different element shapes in the rear lens group, it is important to check the origin of the reduced sensitivity. A good way to test this is to reconstruct the freeform design with even aspherical surfaces and evaluate its performance. Since we are changing pedal surfaces back to aspherical surfaces, we call this reconstructed lens the 'reversed asphere lens'. Fig. 11 shows the lens layout and the nominal MTF performance of the reversed asphere lens and the pedal freeform lens from section 5 . We can see that the freeform lens can be reconstructed to a very similar layout with even aspherical surfaces. The reconstructed system provides the same level of optical performance as the freeform design. The MTF plots for the reversed asphere lens under decenter are provided in Fig. 12. By comparing these MTF plots with the MTF plots of the freeform design in section 5, we realize that despite that the overall construction of the freeform design can be reconstructed using even aspherical surfaces, the detailed surface profile of the freeform design cannot be $100 \%$ reproduced by the aspherical surfaces to achieve the same level of tolerance sensitivity performance. Nevertheless, the tolerance performance of the reversed asphere design did improve compared to the even asphere design in section 5. It seems like using pedal surfaces during the optimization can potentially help the local optimization algorithm in lens design software to find a better solution for even asphere surface designs.

\section{CONCLUSION}

In this paper, we discussed our research in miniature camera lens optimization using freeform surfaces based on the pedal curve to the ellipse. Two patented lenses were used for our evaluation. In section 4, the pedal freeform lens shows better nominal optical performance with smoother OPD plots across the field, and more uniform contrast distribution at high spatial frequencies. The tolerance analysis shows that the pedal freeform lens has lower sensitivity to decenter error compared to the even asphere lens. In section 5, we confirmed our conclusion on decenter sensitivity from section 4 with another evaluation on an even asphere lens, a Q-type polynomial lens and a pedal freeform lens. The pedal freeform lens shows significantly less decenter sensitivity than the other two lenses. On the other hand, there is no obvious difference of decenter sensitivity between the even asphere lens and the Q-type polynomial lens. In section 6, we try to reconstruct the pedal freeform lens in section 5 with even aspherical surfaces. The MTF plots under decenter shows that the low decenter sensitivity property of the pedal freeform surfaces cannot be obtained with even aspherical surfaces. However, by using the reverse of a pedal surface, the optimization software can escape a local minimum and find a better solution using even aspherical surfaces. Overall, we show that by using pedal freeform surfaces, one may increase the nominal optical performance and decrease the surface and element sensitivity to decenter error with less surface parameters. Thus, the pedal curve to the ellipse is useful in miniature imaging lens designs to increase yield.
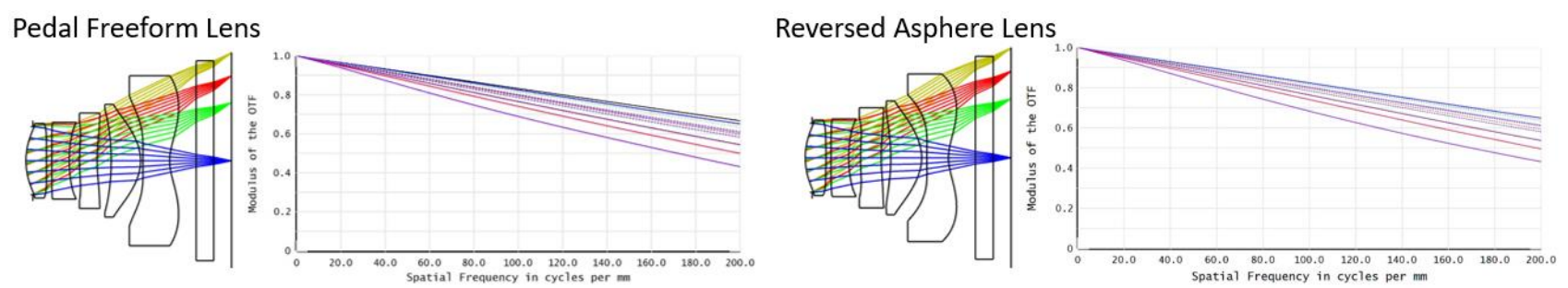

Fig. 11 Layout and nominal MTF plots for pedal freeform lens (left) and reversed asphere lens (right) 


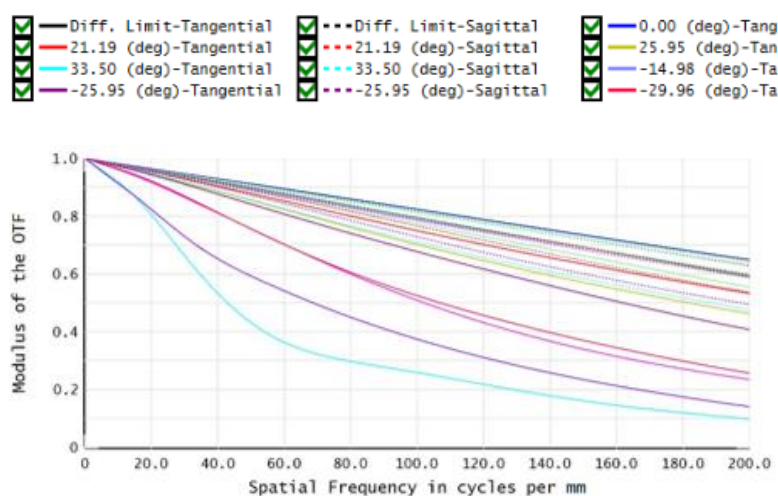

(a)

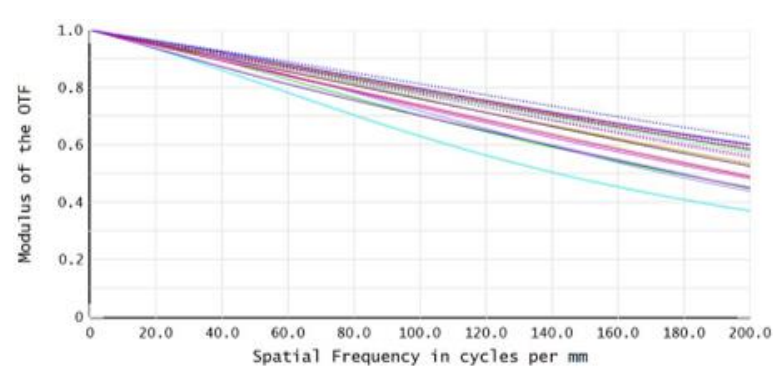

(c)

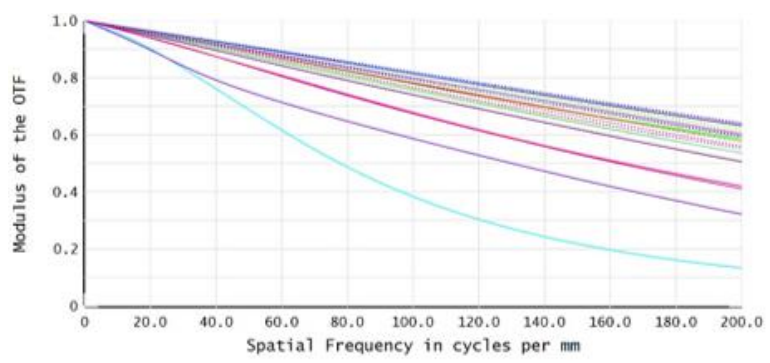

(e)
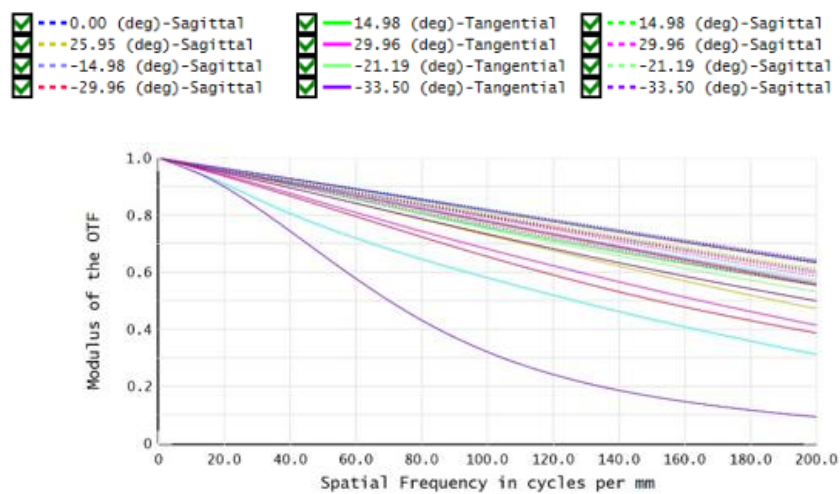

(b)

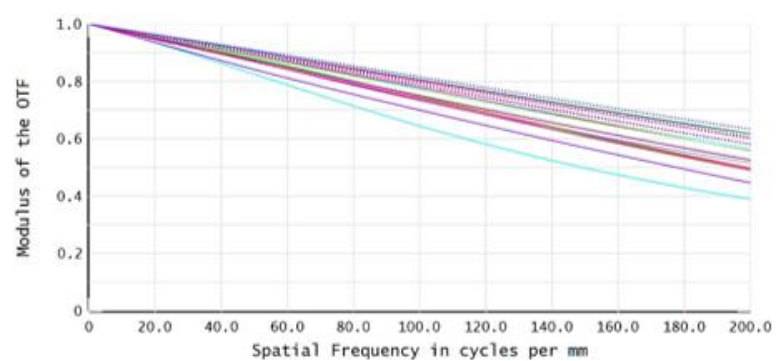

(d)

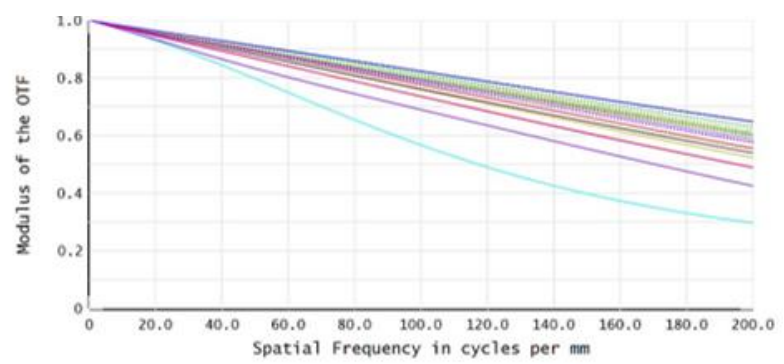

(f)

Fig. 12 MTF plots under 5 micron surface decenter of (a) surface 7, (b) surface 8, (c) surface 9, (d) surface 10, (e) element 4 and (f) element 5.

\section{ACKNOWLEDGEMENT}

The author would like to thank Zemax to provide educational license for OpticStudio. Also thanks to Dmitry Reshidko for writing the user defined surface in OpticStudio.

\section{REFERENCES}

[1] Sasián, J., Reshidko, D. and Li, C., "Aspheric/freeform optical surface description for controlling illumination from point-like light sources," Opt. Eng. 55(11), 115104 (2016).

[2] Clark, P., "Mobile platform optical design," Proc. SPIE 9293, 92931M (2014).

[3] Bareau, J. and Clark, P., "The optics of miniature digital camera modules," Proc. SPIE 6342, 63421F (2006). 
[4] Reshidko, D., and Sasian, J., "Optical analysis of miniature lenses with curved imaging surfaces," Appl. Opt. 54, E216-E223 (2015).

[5] Forbes, G.W., "Shape specification for axially symmetric optical surfaces," Opt. Express 15, 5218-5226 (2007).

[6] Forbes, G.W., "Manufacturability estimates for optical aspheres," Opt. Express 19, 9923-9942 (2011).

[7] Ma, B., Sharma, K., Thompson, K. and Rolland, J., "Mobile device camera design with Q-type polynomials to achieve higher production yield," Opt. Express 21, 17454-17463 (2013). 